- telt worden (Kurve a in Abb. 5). Die Úbereinstimmung der beiden Kurven ist recht gut. Für die $\chi^{-}$ Werte der Familie 0, $u$ würde man in entsprechender Weise die Kurve b der Abb. 5 erwarten. Man erhält aber, wie Kurve b in Abb. 7 zeigt, im allgemeinen wesentlich höhere Werte. Bei der Berechnung der Kurve b in Abb. 5 war man davon ausgegangen, daß die Kerne von $0, u$ in solche Kerne zerfallen $(2, g)$, die vermutlich den Spin Null haben und denen deswegen auch der Wert $\chi=0$ zugeschrieben wurde. Wenn man nun aber nach dem hier genannten Verfahren $\chi$-Werte für $2, g$ ausrechnet, so erhält man die Werte der Kurve c in Abb. 7. Hiernach haben die Kerne $\mathrm{Be}^{10}$, $\mathrm{C}^{14}$, $\mathrm{O}^{18}$ und $\mathrm{Ne}^{22} \chi$-Energien von $2-3 \mathrm{MeV}$. Erst ab $\mathrm{Mg}^{26}$ ist auch $\chi=0$ oder nur wenig von Null verschieden. Die Kurve b in Abb. 5 gibt dann recht gut die Differenzen zwischen den Kurven b und c der Abb. 7 wieder. Die Spinenergie von $\mathrm{C}^{10}$ liegt wohl innerhalb der Fehlergrenze mit auf der Kurve c.

Dieses Resultat ist überraschend, da man bisher annehmen mußte, daß die Kerne 2, $g$ mit $s=0$ auch wirklich die Spinenergie 0 besitzen. Es erhebt sich daher erneut die Frage, ob den Kernen $\mathrm{Be}^{10}, \mathrm{C}^{14}, \mathrm{O}^{18}$ und $\mathrm{Ne}^{22}$ wirklich sowohl die Eigendrehimpuls-Quantenzahl 0 als auch die Bahndrehimpuls-Quantenzahl 0 zuzuordnen ist. Aus der weitgehenden Ahnlichkeit der Kurven für 1, $u$ und $-1, g$ in Abb. 6 b kann man ohne großen Fehler eine $\chi$-Energie für $\mathrm{Al}^{25}$ von $3,6 \pm 0,2 \mathrm{MeV}$ ableiten. Mit diesem Werte ergibt sich aus $\mathrm{Mg}^{24}+p$ ein Wert für die Masse des oben schon vermuteten $\mathrm{Al}^{25}$ mit etwa 8 sec Halbwertszeit von 24,9991 . Die maximal mögliche kinetische Energie der $\beta$ Strahlen wäre dann etwa $3,2 \pm 0,2 \mathrm{MeV}$, durchaus in guter Übereinstimmung mit dem aus Abb. 3 interpolierten Werte von $3,5 \pm 0,3 \mathrm{MeV}$. Für $\mathrm{Al}^{26}$ kann man aus Kurve b der Abb. 7 mit etwas geringerer Genauigkeit einen $\chi$-Wert von $2,3 \pm 0,5 \mathrm{MeV}$ ablesen. Mit Hilfe von $\mathrm{Al}^{26}+n=\mathrm{Al}^{27}$ und unter Benutzung des Wertes von $2,0 \mathrm{MeV}$ für $\mathrm{Al}^{27}$ erhält man für die Masse von $\mathrm{Al}^{26}$ den Wert 25,9933 $\pm 0,5 \mathrm{TME}$. Die maximale $\beta+$-Energie könnte dann $1,9 \pm 0,5 \mathrm{MeV}$ betragen.

\title{
Über die statistische Mechanik regulärer und irregulärer Lösungen
}

\author{
Von Arnold MÜnster \\ Aus dem Chemischen Institut der Universität Heidelberg \\ (Z. Naturforschg. 3 a, 158-172 [1948]; eingegangen am 10. Januar 1948)
}

\begin{abstract}
Es wird eine Methode entwickelt, die unter gewissen Voraussetzungen eine näherungsweise Berechnung der Verteilungsfunktion flüssiger Gemische von beliebig vielen Komponenten beliebiger Molekülgröße und -gestalt ermöglicht. Vorausgesetzt wird, daß 1. eine Komponente, das Lösungsmittel, zu mehr als 50\% der Gesamtmasse vorhanden ist, 2. die Moleküle des Lösungsmittels nicht größer als die der übrigen Komponenten und annähernd kugelförmig sind, 3. zwischen den Molekülen keine weitreichenden Kräfte auftreten. Das Ergebnis wird auf die Probleme der streng regulären binären Lösung sowie der mono- und polydispersen Lösung hochpolymerer Fadenmoleküle angewandt. Im letzteren Falle ergibt sich, daß die thermodynamischen Eigenschaften im allgemeinen von der Verteilung der Polymerisationsgrade abhängen.
\end{abstract}

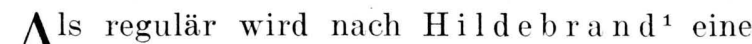
Alösung bezeichnet, deren Verdünnungswärme

$$
\Delta \mathrm{H}_{2} \neq 0
$$

ist, während für die Verdünnungsentropie

$$
\Delta s_{2}=-R \ln v_{2},
$$

$\left(s_{2}=\right.$ Molenbruch des Lösungsmittels LM, $R=$ Gaskonstante) gilt. Dagegen gilt bei irregulären

1 J. S. H i l d e brand, J. Amer. chem. Soc. 51, 66 [1929].
Lösungen die Beziehung (1); es ist aber

$$
\Delta s_{2} \neq-R \ln x_{2} \text {. }
$$

Das Auftreten einer von Null verschiedenen Verdünnungswärme beruht auf zwischenmolekularen Wechselwirkungen, die man gewöhnlich unter dem Begriff der Solvatation zusammenfaßt. In der statistischen Mechanik ist dieselbe dadurch definiert, daß für eine beliebige Konfiguration des Systems die Vertauschung zweier verschiedener Moleküle eine Änderung der potentiel- 
len Energie bewirkt. Da die statistische Berechnung der thermodynamischen Eigenschaften einer Lösung im wesentlichen auf die Ermittlung der Verteilungsfunktion der potentiellen Energie des Gesamtsystems

$$
B(T)=\int \ldots \int \exp (-E / k T) d \tau
$$

( $E=$ potentielle Energie des Gesamtsyștems, $k=$ Boltzmannsche Konstante, $T=$ abs. Temperatur, $d \tau=$ Volumenelement des Konfigurationsraumes) hinausläuft, erkennt man leicht, daß die Berücksichtigung der Solvatation das Problem außerordentlich kompliziert. Während man sich im Falle $\Delta_{H_{2}}=0$ (ideale und athermische Lösung) mit einer bloßen Abzählung der Konfigurationen begnügen kann, muß jetzt eine Integration bzw. Summierung des Ausdrucks exp $(-E / k T)$ über alle Konfigurationen ausgeführt werden. Es lassen sich jedoch Wege zur Lösung der Aufgabe finden, wenn weitreichende Kräfte zwischen den Molekülen ausgeschlossen werden. In diesem Falle setzt sich $E$ additiv aus den Anteilen kleiner Molekülgruppen zusammen; es bleibt dann als wesentliche Aufgabe noch die Abzählung der Konfigurationen, die zu bestimmten Zahlen der ins Auge gefaßten Gruppen gehören.

Auf dieser Grundlage wurde das Problem von $\mathrm{G} u \mathrm{~g}$ g e $\mathrm{nh}$ e i m ${ }^{2}$ für den Spezialfall der sog. streng regulären binären Lösung (strictly regular binary solution) behandelt. Charakteristisch ist für diese Theorie, daß einmal die Energie bereits als in Molekülpaaren additiv angenommen wird, und ferner, daß die Abzählung der zu den verschiedenen Paarzahlen gehörigen Konfigurationen nicht wirklich durchgeführt, sondern durch eine Mittelwertbildung ersetzt wird. Für den Mittelwert stellte Gu g g e $n$ h e i m zunächst rein heuristisch eine Beziehung auf, die wegen ihrer Ähnlichkeit mit dem Massenwirkungsgesetz als quasichemische Gleichung bezeichnet wird. Rus h b r o o k e ${ }^{3}$ zeigte später, daß sich dieselbe (in korrekterer Fassung) mittels des B eth e schen Näherungsverfahrens ${ }^{4}$ exakt begründen läßt. G u g g e n h.e i $\mathbf{m}^{\mathbf{5}}$ selbst gab eine etwas andere Ableitung, die aber im wesentlichen auf dem gleichen Prinzip wie die Bethesche Methode beruht. In dieser Arbeit wurde die Theorie für beliebig viele Komponenten formuliert. Für binäre irreguläre Lösungen von Fadenmolekülen wurde eine Verallgemeinerung der quasi-

2 E. A. G u g g e n h e i m, Proc. Roy. Soc. [London] Ser. A 148, 304 [1935].

3 G. S. R u s h b r o o k e, Proc. Roy. Soc. [London] Ser. A 166, 296 [1938].

${ }_{4}$ H. B e th e, Proc. Roy. Soc. [London] Ser. A 150, 552 [1935].

5 E. A. G u g g e $\mathrm{n}$ h e i m, Proc. Roy. Soc. [London] Ser. A 169, 134 [1939]. chemischen Gleichung nach der Betheschen Methode. von $\mathrm{Orr}^{6}$ entwickelt. G u g g e $\mathrm{nh}$ e i $\mathrm{m}^{7}$ verallgemeinerte die quasichemische Gleichung für Systeme von beliebig vielen Komponenten beliebiger Molekülgrößen. Die Anwendbarkeit dieser Theorie wird aber dadurch eingeschränkt, daß sie einerseits nur für glatte oder verzweigte Ketten, aber nicht für geschlossene Ringe und kompakte Moleküle gilt, andererseits die Gleichungen sich für höhere als binäre Systeme nur in nullter Näherung explizit auswerten lassen.

Die Annahme, daß $E$ in Molekülpaaren additiv ist, setzt voraus, daß das zwischenmolekulare Potential kugelsymmetrisch ist. Es ist leicht einzusehen, daß dies sehr häufig auch nicht an nähernd der Fall ist, selbst wenn die Molekülgestalt nur wenig von der Kugelsymmetrie ábweicht, wie etwa bei $\mathrm{CH}_{3} \mathrm{OH}$. Wird ein solcher Stoff in einem unpolaren LM (Kohlenwasserstoff) gelöst, so besteht eine Tendenz, daß sich möglichst viele polare Gruppen gegenseitig ,,absättigen"; in einem polaren LM ist das Gegenteil der Fall. Ein extremes Beispiel für dieses Verhalten liefern die Seifenmicellen. Die Energie solcher Gruppen kann nicht additiv aus der Wechselwirkungsenergie der Paare berechnet werden, weil dieser Begriff selbst nicht eindeutig definiert ist, sondern von der Orientierung der Moleküle abhängt.

Infolgenden wollen wir versuchen, eine Methode zu entwickeln, welche von den erwähnten Beschränkungen frei ist und gleichzeitig eine direkte Berechnung der Verteilungsfunktion ermöglicht, so daß der Umweg über die ,große Verteilungsfunktion“ (grand partition function) ${ }^{8}$, welche das Dampfdruckgleichgewicht einschließt, entbehrlich wird. Wir werden die Rechnung gleich für eine beliebige Zahl von Komponenten durchführen, was hier keine wesentliche Komplikation bedingt und $\mathrm{zu}$ expliziten Formeln führt.

\section{Die Verteilungsfunktion der potentiellen Energie des Gesamtsystems}

\section{a) Der allgemeine Ansatz}

Wir betrachten eine flüssige Mischung von $i+1$ Komponenten. Eine derselben soll in größerer Menge, sagen wir, zu mehr als $50 \%$ der Gesamtmasse, vorhanden sein. Wir nennen sie das

6 W. J. C. Orr, Trans. Faraday Soc. 40, 320 [1944].

7 E. A. Gu g g e n h e i m, Proc. Roy. Soc. [London] Ser. A 183, 213 [1944].

8 R. H. F ow ler, Proc. Cambridge philos. Soc. 34, 382 [1938]. 
LM und bezeichnen sie mit dem Index 2. Die Moleküle der übrigen $i$ Komponenten, die wir mit den Indizes 11 bis $1 i$ bezeichnen, sollen nicht wesentlich kleiner sein als die des LM, die wir als annähernd kugelförmig voraussetzen; im übrigen ist ihre Größe und Gestalt beliebig. Ferner setzen wir voraus, daß keine weitreichenden Kräfte zwischen den Molekülen auftreten, eine energetische Wechselwirkung also nur zwischen unmittelbar benachbarten Molekülen stattfindet. Noch präziser ist die Formulierung, daß in der Wechselwirkung weiter entfernter Moleküle keine Unterschiede zwischen den verschiedenen Komponenten bestehen (wenn auf „Bausteine“ gleicher Größe reduziert wird) ${ }^{2}$. Die große Verteilungsfunktion eines solchen Systems, das einen Spezialfall der ,kooperativen Gesamtheiten“" darstellt, können wir schreiben

$$
\Xi=\sum_{N_{11}} \ldots \sum_{N_{1 i}} \sum_{N_{2}} \prod_{i} G_{1 i}^{N_{1 i}} G_{2}^{N_{2}} B(T) \prod_{i} \lambda_{1 i}^{* N_{1 i}} \lambda_{2}^{* N_{2}} .
$$

Hier ist $B(T)$ durch Gl. (4) definiert. Die $G$ sind die Verteilungsfunktionen der inneren und kinetischen Energie der Moleküle; sie seien für alle Konfigurationen des Systems gleich. Die $\lambda^{*}$ sind die ,absoluten Aktivitäten" ${ }^{4}$, die mit den chemischen Potentialen durch die Beziehung

$$
\mu=k T \ln \hat{\lambda}^{*}
$$

zusammenhängen, während die gewöhnlichen Aktivitäten $a$ durch die Gleichung

$$
\mu=\mu_{0}(T)+k T \ln a
$$

definiert $\operatorname{sind}^{10}$, wo $\mu_{0}(T)$ eine für jede Temperatur willkürlich gewählte Größe ist. Die $N$ sind die Molekülzahlen, die sich bei der betreffenden Konfiguration in der kondensierten Phase befinden. Da wir die statistische Untersuchung auf die letztere beschränken, benötigen wir die dem thermodynamischen Gleichgewicht entsprechenden Mittelwerte der $N$. Sie ergeben sich aus den Gleichungen

$$
\bar{N}=\xi \frac{\partial \ln \Xi}{\partial \xi},
$$

* In der angelsächsischen Literatur wird der Buchstabe $\lambda$ gebraucht, den wir in der Theorie der athermischen Lösung benötigen. Wir fügen daher zur Unterscheidung einen Stern hinzu.

9 R. H. F o w le r, Statistical Mechanics, 2nd ed., Cambridge 1936. wo

$$
\xi=\lambda^{*} G
$$

ist. Diese Mittelwerte, mit denen wir es im folgenden stets zu tun haben, werden wir von jetzt ab nicht mehr besonders kennzeichnen. Das Problem reduziert sich somit, wie schon bemerkt, auf die Berechnung von $B(T)$ für die obigen Mittelwerte. Wir können die Aufgabe weiter vereinfachen, indem wir die Energie $E$ in passender Weise normieren. Offenbar besitzt $E$ für gewisse Konfigurationen des Systems (wir verstehen darunter jetzt nur noch die kondensierte Phase) ausgeprägte Minima, welche Gleichgewichtslagen der Moleküle entsprechen. Wir nennen sie StandardKonfigurationen ${ }^{11}$ und ihre Energie $E_{\min }$. Die neueren Untersuchungen über die Struktur flüssiger Phasen rechtfertigen die Annahme, daß wir dieselben als verschiedene Besetzungen der Punkte eines quasikristallinen Gitters darstellen können. Streng genommen dürfen wir demselben allerdings nur eine Nahordnung zuschreiben und die Koordinationszahl $z$ nur als einen Mittelwert auffassen. Für das Folgende ist diese Einschränkung zunächst ohne Bedeutung. Die Moleküle des LM werden dann je einen Gitterpunkt besetzen, die übrigen, je nach ihrer Größe, einen oder mehrere. Aus der Gesamtzahl der Standard-Konfigurationen greifen wir nun diejenigen heraus, bei welchen alle gelösten Moleküle nur Moleküle des LM als nächste Nachbarn haben. Alle diese Standard-Konfigurationen besitzen die gleiche Energie $E_{0}$. Für die übrigen setzen wir

$$
E_{\min }=E_{0}+E_{\text {so }} \text {. }
$$

Alle Konfigurationen außerhalb der StandardKonfigurationen können wir als von den letzteren ausgehende zwischenmolekulare Schwingungen auffassen. Die dabei auftretenden Änderungen der Energie der Standard-Konfigurationen hängen, wie schon vor längerer Zeit K. F. $\mathrm{He} \mathrm{r} \mathrm{z} \mathrm{f} \mathrm{e} \mathrm{l} \mathrm{d}{ }^{\mathbf{1 2}}$ bemerkt hat, von der Art der letzteren ab; sie sind daher bei der Berechnung von $B(T)$ zu berücksichtigen. Dieser Gesichtspunkt ist in den bisherigen Untersuchungen vernachlässigt worden. Eine genaue Ermittlung der zugehörigen Verteilungs-

10 G. N. L e w is u. M. R a nd a ll, Thermodyna. mik. Deutsch v. O. Redlich. Wien 1927.

11 R. H. F ow le r u. G. S. R u s h b o o k e, Trans. Faraday Soc. 33, 1272 [1937].

12 K. F. Herzfeld, Kinetische Theorie der Wärme. Müller-Pouillets Lehrbuch der Physik, Bd. III 2, Braunschweig 1925. 
funktionen ist naturgemäß für eine flüssige Phase außerordentlich schwierig. Wir werden uns daher mit einem einfachen quasikristallinen Modell begnügen, welches die Art des Einflusses derselben und die Bedingungen für die bisher benutzte Näherung erkennen läßt. Setzen wir für beliebige Konfigurationen

$$
E=E_{\min }+\Delta E
$$

so können wir schreiben

$$
\begin{aligned}
& B(T)=\exp \left(-E_{0} / k T\right) \\
& . \quad(11) \\
& \int \ldots \int \exp \left[-\left(E_{\mathrm{so}}+\Delta E\right) / k T\right] d \tau .
\end{aligned}
$$

Damit ist die Energie in der gewünschten Weise normiert: wir beziehen von jetzt ab alle Energien auf das Niveau $E_{0}$. Thermodynamisch bedeutet dies, daß wir die unendlich verdünnte Lösung als Normalzustand ${ }^{\mathbf{1 0}}$ für die Änderungen der partialen Wärmeinhalte wählen. Für alle StandardKonfigurationen der Energie $E_{0}$ hat auch $\Delta E$ den gleichen Wert, der mit $\Delta E_{0}$ bezeichnet werde, während wir für die übrigen jetzt $\Delta E_{s}$ schreiben wollen. Dann kann Gl. (11) geschrieben werden

$$
\begin{aligned}
B(T)=\exp & \left(-E_{0} / k T\right) \\
& \left\{f \ldots \int \exp \left(-\Delta E_{0} / k T\right) d \tau\right. \\
& +\int_{\left(\tau_{s}\right)} \ldots f\left[\exp \left[-\left(E_{\mathrm{so}}+\Delta E_{s}\right) / k T\right]\right. \\
& \left.\left.-\exp \left(-\Delta E_{0} / k T\right)\right] d \tau\right\},
\end{aligned}
$$

wo $\tau_{s}$ das Gebiet des Konfigurationsraumes bezeichnet, in welchem $E_{s 0} \neq 0$ ist. Das erste der beiden Integrale enthält die reine (athermische) Lagenstatistik des Systems, das zweite den Beitrag der Solvatation zu $B(T)$. Zur Berechnung der Verteilungsfunktion der athermischen Lösung sind verschiedene Methoden entwickelt worden. Wir erwähnen die filling-up-Methode, die schon Fow le r und Rushbrooke ${ }^{11}$ einführten und die später von $\mathrm{Fl}$ o r $\mathrm{y}^{13}, \mathrm{Hu}$ g g i n s ${ }^{14}$ und kürzlich wieder von G. V. Schulz ${ }^{15,16,17}$ benutzt wurde, das B ethe sche Näherungsverfahren *, das zuerst von $\mathrm{Ch}$ an $\mathrm{g}^{18}$ auf zweidimensionale

13 P. J. Flo r y, J. chem. Physics 10, 51 [1942].

14 M. L. Hu g g in s, J. physic. Chem. 46, 151. [1942].

15 G. V. S chulz, Z. Naturforschg. 2a, 27 [1947].

16 G. V. S c hul z, Z. Naturforschg. 2a, 348 [1947].

${ }_{17}$ G. V. S ch u z , Z. Naturforschg. 2a, 411 [1947].

18 T. S. Ch ang, Proc. Roy. Soc. [London] Ser. A 169, 512 [1939].
Probleme, dann von Mille r ${ }^{\mathbf{1 9}, 20}$ auf dreidimensionale angewandt wurde, und schließlich die Methode der virtuellen Moleküle ${ }^{21,22}$. Da wir die letztere für die weitere Rechnung benötigen, wollen wir sie kurz rekapitulieren. Eine eingehendere Diskussion der Verhältnisse bei höheren Konzentrationen wird demnächst erfolgen.

b) Die Zahl der Standard-Konfigurationen der athermischen Lösung

Bei einer idealen Lösung kann man die Zahl der Standard-Konfigurationen bekanntlich in der Weise ermitteln, daß man alle möglichen Vertauschungen zwischen den Molekülen vornimmt. Dieses einfache Rezept versagt, wenn die verschiedenen Moleküle nicht mehr die gleiche Zahl von Gitterpunkten besetzen. Man kann sich aber helfen, indem man aus den Molekülen des LM und $i-1$ gelöster Komponenten Gebilde konstruiert, die nach Größe und Gestalt denen der $i$-ten Komponente gleichen und daher mit ihnen vertauscht werden können. Wir nennen sie virtuelle Moleküle; ihre Zahl sei $\Lambda$ für die $i$-te Komponente. Dann wird die Zahl der Standard-Konfigurationen

$$
Q=\Pi_{i} \frac{\left(N_{1 i}+\Lambda_{i}\right) ! N_{2} !}{\Lambda_{i} !} .
$$

Die Funktionen $\Lambda_{i}$ hängen von der Größe, Gestalt, Beweglichkeit'und Konzentration aller Komponenten ab. Nach ihrer Definition sind sie gleich der Zahl der Anordnungsmöglichkeiten für ein Molekül der $i$-ten Komponente im Gitter der Lösung, vermindert um diejenigen, die ganz oder teilweise auf Gitterpunkte fallen, die bereits von Molekülen der $i$ gelösten Komponenten besetzt sind (soweit diese nicht selbst den Teil eines virtuellen Moleküls bilden). Um die virtuellen Moleküle abzählen zu können, müssen wir übereinkommen, daß sie jeweils von einem Gitterpunkt aus nach einer festgelegten Vorschrift konstruiert werden. Die Zahl der sich dabei ergebenden Möglichkeiten sei $f(z)_{i}$ (G u g g e $\mathrm{n}$ h e i m ${ }^{23}$ bezeichnet diese Größe mit $\varrho_{i}$ ). Die durch Anwesenheit reel-

19. A. R. M i l l e r, Proc. Cambridge philos. Soc. 38, 109 [1942].

20 A. R. M i ll e r, Proc. Cambridge philos. Soc. 39, 54 [1943].

21 A. M ü n s t e r, Kolloid-Z. 105், 1 [1943].

22 A. M ü nster, Z. Naturforschg. 1, 311 [1946].

23 E. A. Gu g g e $\mathrm{n}$ h e i m, Proc. Roy. Soc. [London] Ser. A 183, 203 [1944]. 
ler Moleküle ausfallenden virtuellen Moleküle können wir einteilen in solche, bei denen der Ausgangs-Gitterpunkt, und in solche, bei denen einer der darauf folgenden Gitterpunkte besetzt ist. Der letztere Anteil bedeutet, daß in der Umgebung eines reellen gelösten Moleküls (sofern dieses mehrere Gitterpunkte besetzt) ein Gebiet existiert, in welchem sich andere gelöste Moleküle überhaupt nicht oder nur mit bestimmten Orientierungen aufhalten können. Wir bezeichnen diese Tatsache als Störungseffekt ${ }^{16,22}$. Für verdünnte Lösungen von Fadenmolekülen überwiegt er den ersten Anteil bei weitem. Nach dem Vorstehenden können wir für $\Lambda_{i}$ ansetzen

$$
\begin{aligned}
A_{i}=( & \left.N_{2}+\sum_{i^{\prime}} n_{i^{\prime}} N_{1 i^{\prime}}\right) f(z)_{i}-\left[\sum_{i^{\prime}} n_{i^{\prime}} Q_{i^{\prime}} N_{1 i^{\prime}}\right. \\
& \left.+\frac{1}{2} \sum_{i^{\prime}} a_{i i^{\prime}}\left(N_{11}, \ldots N_{1 i}, N_{2}\right) n_{i^{\prime}} N_{1 i^{\prime}}\right] f(z)_{i} .
\end{aligned}
$$

Hier ist $n_{i}$ die Zahl der Gitterpunkte, die ein Molekül der $i$-ten Komponente besetzt, $\varrho_{i^{\prime}}$ ein Reduktionsfaktor, der die Tatsache berücksichtigt, daß auch Moleküle der $i-1$ übrigen gelösten Komponenten Teile virtueller Moleküle sein können. Für streng reguläre Lösungen (s. II b) , ist $\varrho_{i^{\prime}}=0^{24}$; bei verdünnten Lösungen hochpolymerer Fadenmoleküle kann man mit $\varrho_{i^{\prime}}=1$ rechnen ${ }^{22}$. $\alpha_{i i^{\prime}}\left(N_{11}, \ldots N_{1 i}, N_{2}\right)$ ist eine Funktion der Molekülzahlen sämtlicher Komponenten, die durch die Größe, Gestalt und Beweglichkeit der gelösten Moleküle bestimmt wird. Für sehr verdünnte Lösungen nimmt sie einen Grenzwert an, den wir früher ${ }^{22}$ als Störungsparameter bezeichnet haben. Derselbe werde im folgenden einfach mit $\alpha_{i i^{\prime}}$ bezeichnet. Für die weitere Rechnung ist es zweckmäßig, noch zwei Größen zu definieren, den virtuellen Molenbruch der Komponente $i$

$$
\gamma_{i}=\frac{N_{1 i}}{N_{1 i}+\Lambda_{i}}
$$

und eine Größe

$$
N_{1 i}=\frac{N_{1 i}}{N_{2}+\sum_{i} n_{i} N_{1 i}} .
$$

Für die streng reguläre Lösung gehen beide in den gewöhnlichen Molenbruch über.

${ }^{24}$ Nur für die Komponente $i$, auf die sich $\Lambda_{i}$ bezieht, ist stets $\varrho_{i}=1$. c) Allgemeine Formulierung der Verteilungsfunktion

Die Integrale der Gl. (12) ląssen sich allgemein in der Weise berechnen, daß für jede StandardKonfiguration über die zwischenmolekularen Schwingungen integriert und dann über alle Standard-Konfigurationen summiert wird. Das zweite Integral kann dann geschrieben werden

$$
\begin{aligned}
& \int_{\left(\tau_{s}\right)} \ldots \int\left\{\exp \left[-\left(E_{\text {so }}+\Delta E_{s}\right) / k T\right]\right. \\
& \left.-\exp \left(-\Delta E_{0} / k T\right)\right\} d \tau \\
& =\sum_{\left(\tau_{s}\right)}\left[\exp \left(-E_{\mathrm{so}} / k T\right) \int \ldots \int \exp \left(-\Delta E_{s} / k T\right) d \sigma\right. \\
& \left.-\int \ldots \int \exp \left(-\Delta E_{0} / k T\right) d \sigma\right]
\end{aligned}
$$

wo $d \sigma$ das Volumenelement des ,Schwingungsraumes" einer Standard-Konfiguration bezeichnet. Wir nehmen an, daß sich die Verteilungsfunktion der zwischenmolekularen Schwingungen für jede Standard-Konfiguration in $N_{2}+\Sigma_{i} n_{i} N_{1 i}$ Faktoren $\Gamma$ separieren läßt. Für jede Standard-Konfiguration der Energie $E_{0}$ können wir alle $\Gamma$ als gleich ansehen; diese Größen mögen mit $\Gamma_{1}$ bezeichnet werden. Im Gebiet $\tau_{s}$ ist ein Teil der Faktoren $\Gamma \neq \Gamma_{1}$. Die Zahl derselben (die für die einzelnen Standard-Konfigurationen des Gebietes $\tau_{s}$ verschieden ist) sei jeweils $\omega$. Dann wird aus Gl. (12)

$$
B(T)=\exp \left(-E_{0} / k T\right) \Gamma_{1}^{N_{2}+\sum_{i} n_{i} N_{1 i}}(Q+Z),
$$

wo

$$
Z=\sum_{\left(\tau_{s}\right)}\left[\exp \left(-E_{\mathrm{so}} / k T\right)\left(I / I_{1}\right)^{\omega}-1\right]
$$

ist.

Das Gebiet $\tau_{s}$ umfaßt alle Standard-Konfigurationen, bei welchen „Gruppen“ gelöster Moleküle auftreten, d.h. gelöste Moleküle direkt benachbarte Gitterpunkte besetzen. Wir ordnen diese Gruppen nach Art und Zahl der darin vorkommenden Moleküle in Typen $r$; es seien $l_{r}$ Gruppen vom Typus $r$ bei gegebener Standard-Konfiguration vorhanden. In einer solchen Gruppe mögen $t_{i r}$ Moleküle der Komponente $i$ vertreten sein. Damit geben wir der Gl. (19) die Form

$$
\begin{aligned}
Z= & \sum g_{l_{r}} \sum_{v_{1_{r}}} \ldots \sum_{v_{l_{r}}} \ldots \Sigma \ldots \Sigma \\
& \cdot\left\{\exp \left[-\Sigma\left(\Sigma E_{l_{r}}^{*}\right) / k T\right] \prod_{r}\left(\Gamma_{r} / \Gamma_{1}\right)^{\sum_{r}^{l_{m_{r}}}}-1\right\} .
\end{aligned}
$$


Hier ist $g_{l_{r}}$ die Zahl der Möglichkeiten, eine Konfiguration herzustellen, welche $l_{r}$ Gruppen vom Typus $r$ enthält. Es ist $r$-fach über alle $l_{r}$ zu summieren. Die folgende Summierung geht $\sum_{r} l_{r^{-}}$ fach über alle je $\nu$ Konfigurationen der einzelnen Gruppen. Das Summenzeichen in der runden Klammer erstreckt sich über die $l_{r}$-Gruppen vom Typus $r$, das vor der runden Klammer über alle $r$. $E_{l_{r}}^{*}$ ist die Energie der $l_{r}$-ten Gruppe vom Typus $r, m_{r}$ die Zahl der Faktoren $\Gamma_{r} \neq \Gamma_{1}$ für eine Gruppe vom Typus $r$. Die Größen $g_{l_{r}}$ sind in Wirklichkeit Mittelwerte über die je $\nu$ Konfigurationen der Gruppen. Ihre Einführung stellt das erste Näherungsprinzip der Theorie dar.

$g^{\prime}{ }_{r}$ läßt sich in zwei Faktoren zerlegen: die Zahl der Herstellungsmöglichkeiten für $l_{r}$ Gruppen vom Typus $r$ aus $\Sigma_{i} N_{1 i}$ Molekülen und den ..Lösungsfaktor", welcher die zugehörigen Konfigurationen der Gesamtlösung angibt. Der erste Faktor ist

$$
\frac{\prod_{i} N_{1 i} !}{\prod_{i}\left(N_{1 i}-\sum_{r} t_{i, r} l_{r}\right) ! \prod_{r} l_{r} ! \prod_{r} \sigma_{r}^{l_{r}}}
$$

wo $\sigma_{r}$ die Symmetriezahl einer Gruppe vom Typus $r$ ist. Der Lösungsfaktor lautet, wenn wir die Gruppen als eigene Molekülarten einführen und berücksichtigen, daß ihre Zahl bei allen Vertauschungen konstant gehalten werden muß,

$$
\prod_{i} \frac{\left(N_{1 i}-\sum_{r} t_{i r} l_{r}+\Lambda_{i}^{\prime}\right) !}{\Lambda_{i}^{\prime} !} \prod_{r} \frac{\left(l_{r}+\Lambda_{r}^{\prime}\right) !}{\Lambda_{r}^{\prime} !} N_{2} !
$$

Der Strich an den $\Lambda^{\prime}$ bedeutet, daß diese für konstante $l_{r}$ zu bestimmen sind, d. h. die den reellen Molekülen benachbarten Gitterpunkte dürfen bei den Vertauschungen nicht besetzt werden. Bei der Summierung über den Klammerausdruck der Gl. (20) ist zu beachten, daß bei gegebenen $l_{\text {r }}$ nur solche Konfigurationen berücksichtigt werden, für welche sämtliche $E_{l_{r}}^{*} \neq 0$ sind, damit keine mehrfachen Zählungen auftreten. Man erhält dann für den ganzen Faktor

$$
\prod_{r}\left\{\sum_{\nu_{r}}\left[\exp \left(-E_{r}^{*} / k T\right)\left(I_{r} / \Gamma_{1}\right)^{m_{r}}-1\right]\right\}^{l_{r}} \equiv \prod_{r} \Phi_{r}^{l_{r}}
$$

wenn $\Phi_{r}$ die ,lokale Verteilungsfunktion“ einer Gruppe vom Typus $r$ bezeichnet. Der allgemeine Ausdruck für die Funktion $Z$ lautet somit

$$
Z=\Sigma\left\{\prod_{i} \frac{\left(N_{1 i}-\sum_{r} t_{i r} l_{r}+\Lambda_{i}^{\prime}\right) !}{\Lambda_{i}^{\prime} !} \prod_{r} \frac{\left(l_{r}+\Lambda_{r}^{\prime}\right) !}{\Lambda_{r}^{\prime} !} N_{2} ! \frac{\prod_{i} N_{1 i} !}{\prod_{i}\left(N_{1 i}-\sum_{r} t_{i r} l_{r}\right) ! \prod_{r} l_{r} ! \prod_{r} \sigma_{r}^{l_{r}}} \prod_{r} \Phi_{r}^{l_{r}}\right\} .
$$

Wir betrachten zunächst den Lösungsfaktor. Auf Grund der Voraussetzung sind die Fakultätenausdrücke stets große Zahlen. Wir können daher die Stirlingsche Formel anwenden und erhalten für den Faktor:

$$
\begin{array}{r}
\Pi_{i} \exp \left\{\left(N_{1 i}-\sum_{r} t_{i r} l_{r}\right) \ln \left(N_{1 i}^{\prime}-\sum_{r} t_{i r} l_{r}+\Lambda_{i}^{\prime}\right)+\Lambda_{i}^{\prime} \ln \left[\left(N_{1 i}-\sum_{r} t_{i r} l_{r}+\Lambda_{i}^{\prime}\right) / \Lambda_{i}^{\prime}\right]-\left(N_{1 i}-\sum_{r} t_{i r} l_{r}\right)\right\} \\
\cdot \prod_{r} \exp \left\{l_{r} \ln \left(l_{r}+\Lambda_{r}^{\prime}\right)+\Lambda_{r}^{\prime} \ln \left[\left(l_{r}+\Lambda_{r}^{\prime}\right) / \Lambda_{r}^{\prime}\right]-l_{r}\right\} \exp \left\{N_{2} \ln N_{2}-N_{2}\right\} .
\end{array}
$$

Dieser Ausdruck ist nicht ohne weiteres verwendbar. Mit seiner Umformung führen wir das zweite Näherungsprinzip ein. Es besteht im wesentlichen darin, daß wir bei der Abzählung der Konfigurationen für gegebene $l_{r}$ die Bedingung konstanter $l_{r}$ nur für die Zahl der statistischen Individuen, aber nicht für den Störungseffekt berücksichtigen. Die analytischen Bedingungen dafür lauten

$\ln \frac{N_{1 i}+\Lambda_{i}}{N_{1 i}-\sum_{r} t_{i r} l_{r}+\Lambda_{i}^{\prime}} \ll \ln \left(N_{1 i}-\sum_{r} t_{i r} l_{r}+\Lambda_{i}^{\prime}\right)$

Für verdünnte Lösungen ist die Gültigkeit dieser Beziehungen ohne weiteres ersichtlich. Um die Verhältnisse bei höheren Konzentrationen zu überblikken, schreiben wir zunächst den expliziten Ausdruck für $\Lambda_{i}^{\prime}$ an. Er lautet, wenn $q_{i}$ die mittlere Zahl der freien (d. h. von LM-Molekülen besetzten) Gitternachbarn eines Moleküls der Komponente $i$ für ge- 
gebene $l_{r}$ ist und über die Konfigurationen der Gruppen gemittelt wird,

$$
\begin{array}{r}
\Lambda_{i}^{\prime}=\left(N_{2}+\sum_{i^{\prime}} n_{i^{\prime}} N_{1 i^{\prime}}\right) f(z)_{i}-\left[\sum_{i^{\prime}}\left(n_{i^{\prime}}+q_{i^{\prime}}\right) Q_{i^{\prime}}^{\prime} N_{1 i^{\prime}}+\frac{1}{2} \sum_{i^{\prime}} \alpha_{i i^{\prime}}^{\prime}\left(N_{11}-\sum_{r} t_{1 r} l_{r}, \ldots N_{1 i}-\sum_{r} t_{i r} l_{r}, N_{2}, l_{r}\right)\right. \\
\left.\cdot n_{i^{\prime}}\left(N_{1 i^{\prime}}-\underset{r}{\sum} t_{i^{\prime} r} l_{r}\right)+\frac{1}{2} \sum_{r} a_{i r}^{\prime}\left(N_{11}-\sum_{r} t_{1 r} l_{r}, \ldots N_{1 i}-\sum_{r} t_{i r} l_{r}, N_{2}, l_{r}\right) n_{r} l_{r}\right] f(z)_{i}, \quad(26)
\end{array}
$$

wo die $\varrho^{\prime}$ und $\alpha^{\prime}(\ldots)$ für konstante $l$, bestimmt sind. Man sieht sofort, daß für große kompakte Moleküle $n_{i} \gg q_{i}$ und $\alpha_{i i^{\prime}}^{\prime}(\ldots) \approx \alpha_{i i^{\prime}}(\ldots)$ ist. Schließlich ist bei höheren Konzentrationen $\alpha_{i r}^{\prime}(\ldots) \approx \sum_{i^{\prime}} c_{i i^{\prime}}^{\prime} t_{i^{\prime} r}$, so daß man unbedenklich (23) als erfüllt ansehen kann. Bei Fadenmolekülen liegen die Verhältnisse insofern ungünstiger, als hier in verdünnter Lösung $q_{i}>n_{i}$ ist. Da jedoch mit wachsender Konzentration im Mittel $l_{r}$ und $t_{i r}$ wachsen, nimmt $q_{i}$ im Mittel ab. Hier gilt ferner

$$
a_{i i^{\prime}}(\ldots)<a_{i i^{\prime}}^{\prime}(\ldots)<(z-2) \alpha_{i i^{\prime}}(\ldots),
$$

andererseits aber

$$
a_{i r}^{\prime}(\ldots)<\sum_{i^{\prime}} \alpha_{i i^{\prime}}^{\prime}(\ldots) t_{i^{\prime} r},
$$

so daß eine wenigstens teilweise Kompensation stattfindet. Schließlich ist zu beachten, daß, wie demnächst gezeigt wird, die $\alpha(\ldots)$ mit wachsender Konzentration stark abnehmen. Die Verwendung von (23) erscheint daher auch für diesen Fall gerechtfertigt. Bei einem Gemisch von Monomeren verschwinden alle $\alpha_{i i^{\prime}}(\ldots)$, die $\alpha_{i i^{\prime}}^{\prime}(\ldots)$ und $\alpha_{i r}^{\prime}(\ldots)$ sind relativ klein, ferner ist $n_{i}=1$ und in verdünnten Lösungen $q_{i}=z$. In konzentrierteren Lösungen nimmt dieser Wert wieder stark ab, so daß man mit (23) rechnen kann. (24) beruht auf einer mit dem quadratischen Glied abbrechenden Reihenentwicklung des zweiten Logarithmus (das lineare Glied verschwindet wegen des folgenden Summanden). Bei Hochpolymeren unterliegt dieses Verfahren und die Gültigkeit von (24) keinen Bedenken, da hier in dem betrachteten Konzentrationsbereich stets $N_{1 i} \ll N_{2}$ ist. Bei Monomeren gilt dies, wenn man $N_{1 i}-\sum_{r} t_{i r} l_{r} \ll N_{1 i}$ voraussetzen kann, was bei höheren Konzentrationen für die Mehrzahl der Konfigurationen zutrifft. Die Gültigkeit von (25) beruht darauf, daß notwendig $l_{r} \ll N_{1 i}$ ist.

Es kann somit gesagt werden, daß die Gültigkeit der obigen Voraussetzungen über den Bereich der verdünnten Lösung hinausgeht. Eine allgemeine Grenze dafür kann jedoch naturgemäß nicht angegeben werden. Diese Frage kann nur für den konkreten Einzelfall entschieden werden.

Auf Grund der Voraussetzungen (23), (24), (25) können wir für den Lösungsfaktor schreiben $\exp \left\{\sum_{i} N_{1 i} \ln \left(N_{1 i}+\Lambda_{i}\right)+\sum_{i} \Lambda_{i} \ln \left[\left(N_{1 i}+\Lambda_{i}\right) / \Lambda_{i}\right]\right.$

Damit wird

$$
\left.+N_{2} \ln N_{2}-\sum_{i} N_{1 i}-N_{2}\right\} \prod_{i}\left(N_{1 i}+\Lambda_{i}\right)^{-\sum_{r} t_{i r} l_{r}} \prod_{r} \Lambda_{r}^{\prime^{l} r} .
$$

$$
\begin{aligned}
Z=\exp \left\{\sum_{i} N_{1 i} \ln \left(N_{1 i}+\Lambda_{i}\right)\right. & +\sum_{i} \Lambda_{i} \ln \left[\left(N_{1 i}+\Lambda_{i}\right) / \Lambda_{i}\right] \\
& \left.+N_{2} \ln N_{2}-\sum_{i} N_{1 i}-N_{2}\right\} \sum_{\prod_{i}\left(N_{1 i}-\sum_{r} t_{i r} l_{r}\right) ! \prod_{r} l_{r} ! N_{0} \sum_{r}\left(\sum_{i}^{\left.z_{i r}-1\right) l_{r}}\right.}^{\prod_{i} N_{1 i} ! \prod_{r} \varkappa_{r}^{l_{r}}} .
\end{aligned}
$$

Hier ist in jedem Summanden unter dem Summenzeichen wenigstens ein $l_{r} \neq 0$ und

$$
\varkappa_{r}=\frac{N_{0}^{\sum_{i} t_{i r}-1} \Lambda_{r}^{\prime}}{\sigma_{r} \prod_{i}\left(N_{1 i}+\Lambda_{i}\right)^{t_{i r}}} \Phi_{r} .
$$

$N_{0}$ ist eine willkürliche Konstante der Größenordnung $N_{2}$, für welche wir die Loschmidtsche Zahl setzen können. Führen wir Gl. (28) und die mit Benutzung der Stirlingschen Formel umgeformte Gl. (13) in Gl. (18) ein, so folgt 


$$
\begin{aligned}
B(T)=\exp \left(-E_{0} \mid k T\right) \Gamma_{1}{ }^{N_{2}+\sum_{n_{i} N_{1 i}}} \exp \left\{\sum_{i} N_{1 i} \ln \left(N_{1 i}+\Lambda_{i}\right)+\sum_{i} \Lambda_{i} \ln \left[\left(N_{1 i}+\Lambda_{i}\right) / \Lambda_{i}\right]\right. \\
\left.\quad+N_{2} \ln N_{2}-\sum_{i} N_{1 i}-N_{2}\right\} \sum \frac{\prod_{i} N_{1 i} ! \prod_{r} x_{r}^{l_{r}}}{\prod_{i}\left(N_{1 i}-\sum_{r} t_{i r} l_{r}\right) ! \prod_{r} l_{r} ! N_{0} \sum_{r}\left(\sum_{i} t_{i r}-1\right) l_{r}}
\end{aligned}
$$

Dabei sind jetzt im ersten Summanden alle $l_{r}=0$. Wir setzen nun

$$
\sum \frac{\prod_{i} N_{1 i} ! \prod_{r} x_{r}^{l_{r}}}{\prod_{i}\left(N_{1 i}-\sum_{r} t_{i r} l_{r}\right) ! \prod_{r} l_{r} ! N_{0}^{\sum_{r}\left(\sum_{i} t_{i r}-1\right) l_{r}}}=\exp \left(N_{0} g\right)
$$

und erhalten als allgemeinen Ausdruck für die Verteilungsfunktion der potentiellen Energie des Gesamtsystems

$$
\begin{aligned}
& B(T)=\exp \left(-E_{0} / k T\right) \dot{\Gamma}_{1}^{N_{2}+\sum_{i} n_{i} N_{1 i}} \exp \left\{\sum_{i} N_{1 i} \ln \left(N_{1 i}+\Lambda_{i}\right)+\sum_{i} \Lambda_{i} \ln \left[\left(N_{1 i}+\Lambda_{i}\right) / \Lambda_{i}\right]\right. \\
& \left.+N_{2} \ln N_{2}-\sum_{i} N_{1 i}-N_{2}\right\} \exp \left(N_{0} g\right) \text {. }
\end{aligned}
$$

d) Die Ermittlung der Funktion $g$

Um einen analytischen Ausdruck für die Funktion $g$ zu finden, bedienen wir uns einer Methode, die von $\mathrm{Ursel}^{25}$ in die Gastheorie eingeführt wurde und die wir für beliebig viele Komponenten verallgemeinern ${ }^{\mathbf{2}}$. Die Benutzung dieser Methode stellt das dritte Näherungsprinzip unserer Rechnung dar.

Wir führen zunächst für jedes $N_{1 i}$ eine Hilfsvariable $y_{i}$ ein und schreiben die Summe der Gl. (30)

$F\left(x_{r}, y_{i}\right)$

$=\sum \frac{\Pi\left(y_{i} N_{0}\right)^{N_{1 i}-\sum_{r} t_{i r} l_{r}} \prod_{r}\left(\varkappa_{r} N_{0}\right)^{l_{r}} \prod_{i}\left(N_{1 i} !\right) N_{0}^{-\sum_{i} N_{1 i}}}{\prod_{i}\left(N_{1 i}-\sum_{r} t_{i r} l_{r}\right) ! \prod_{r} l_{r} !}$.
Wir suchen die Werte dieser Funktion für alle $y_{i}=1$. Es ist zunächst

$$
\frac{1}{N_{0}} \frac{\partial F}{\partial x_{r}}=\prod_{i}\left(\frac{1}{N_{0}} \frac{\partial}{\partial y_{i}}\right)^{t_{i r}} F
$$

Wir setzen

$$
y_{i}=\exp z_{i}
$$

und führen den Operator

$$
\delta_{i}=\sum_{r} t_{i r} x_{r} \frac{\partial}{\partial x_{r}}
$$

ein. Dann ist

$$
\left(\frac{\partial}{\partial z_{i}}+\delta_{i}\right)^{k} F=N_{1 i}^{k} F
$$

Aus Gl. (33) und (34) erhält man

$$
\frac{1}{N_{0}} \frac{\partial F}{\partial x_{r}}=\exp \left(-\sum_{i} t_{i r} z_{i}\right) \prod_{i}\left(\frac{1}{N_{0}} \frac{\partial}{\partial z_{i}}-\frac{t_{i r}-1}{N_{0}}\right) \ldots\left(\frac{1}{N_{0}} \frac{\partial}{\partial z_{i}}-\frac{1}{N_{0}}\right) \frac{1}{N_{0}} \frac{\partial}{\partial z_{i}} F .
$$

Aus Gl. (36) und (37) folgt

$$
\frac{1}{N_{0}} \frac{\partial F}{\partial x_{r}}=\exp \left(-\sum_{i} t_{i r} z_{i}\right) \prod_{i}\left(\frac{N_{1 i}}{N_{0}}-\frac{t_{i r}-1}{N_{0}}-\frac{\delta_{i}}{N_{0}}\right) \cdots\left(\frac{N_{1 i}}{N_{0}}-\frac{1}{N_{0}}-\frac{\delta_{i}}{N_{0}}\right)\left(\frac{N_{1 i}}{N_{0}}-\frac{\delta_{i}}{N_{0}}\right) F .
$$

Setzen wir jetzt alle $y_{i}=1$ bzw. alle $z_{i}=0$ und benützen Gl. (30), so wird

$\frac{\partial g}{\partial \varkappa_{r}}=\prod_{i}\left(\frac{N_{1 i}}{N_{0}}-\frac{t_{i r}-1}{N_{0}}-\delta_{i} g-\frac{\delta_{i}}{N_{0}}\right) \cdots\left(\frac{N_{1 i}}{N_{0}}-\frac{1}{N_{0}}-\delta_{i} g-\frac{\delta_{i}}{N_{0}}\right)\left(\frac{N_{1 i}}{N_{0}}-\delta_{i} g\right)$,

25 H. D. Ursell, Proc. Cambridge philos. Soc. 23, 685 [1927].
${ }_{26}$ Für zwei Komponenten findet sich die Rechnung bei $\mathrm{Ur}^{2}$ sell ${ }^{25}$. 
wo die $\delta_{i}$ auf alle rechts von ihnen stehenden Funktionen wirken. Wir vernảchlässigen jetzt die Summanden $\left(t_{i r}-1\right) / N_{0}$ usw. und $\delta_{\imath} / N_{0}$. Dann folgt

$$
\frac{\partial g}{\partial \varkappa_{r}}=\prod_{i}\left(\frac{N_{1 i}}{N_{0}}-\delta_{i} g\right)^{t_{i r}} .
$$

Wir definieren jetzt eine neue Größe

$$
h_{i}=\frac{N_{1 i}}{N_{0}}-\delta_{i} g .
$$

Dann ist nach Gl. (35) und (40)

$$
h_{i}=\frac{N_{1 i}}{N_{0}}-\sum_{r} t_{i r} \varkappa_{r} \Pi_{i} h_{i}{ }^{{ }_{i r}},
$$

und wir erhalten

$$
\frac{\partial g}{\partial \varkappa_{r}}=\prod_{i} h_{i}{ }^{t_{i r}} .
$$

Diese partiellen Differentialgleichungen sind unter der Voraussetzung (42) für die Randbedingung $g=0, x_{r}=0$ zu lösen. Die Randbedingung formuliert die bereits früher ${ }^{27}$ in der thermodynamischen Theorie der Solvatation benutzte Tatsache, daß für $T \rightarrow \infty$ die Solvatation verschwindet. Die Lösung wird in der Weise durchgeführt, daß man zunächst aus Gl. (42) die $h_{i}$ in sukzessiver Näherung als Reihe berechnet und jeweils diese Werte in die Gl. (44) einsetzt. Damit ist gezeigt, daß die Darstellung Gl. (30) mit beliebiger Näherung möglich ist.

\section{e) Der Gültigkeitsbereich der Theorie}

Da nach den Ergebnissen des vorhergehenden Abschnittes die hier entwickelte Methode das betrachtete Problem nicht in geschlossener Form, sondern als eine nach Potenzen der Konzentration fortschreitende Reihenentwicklung löst, ist es zweckmäßig, hier kurz die Frage nach dem Gültigkeitsbereich der Theorie zu erörtern. Soweit es sich dabei um die Benutzung des Gittermodells handelt, ist dieses Problem kürzlich von $\mathrm{Orr}^{6}$ diskutiert worden; wir wollen daher an dieser Stelle nicht darauf eingehen, sondern uns auf die Frage beschränken, für welchen Konzentrationsbereich die Theorie anwendbar ist und wieweit

${ }_{27}^{27}$ A. II ü nster, Z. Naturforschg. 2a, 284 [1947]. man das beschriebene Näherungsverfahren fortzusetzen hat.

Am Anfang wurde festgesetzt, daß die als LM bezeichnete Komponente zu mehr als $50 \%$ der Gesamtmasse vorhanden sein soll. In I c haben wir diese Voraussetzung zur Rechtfertigung einiger wesentlicher Vereinfachungen benutzt. Trotzdem sind die Gründe dieser Beschränkung primär nicht mathematischer, sondern physikalischer Natur. Die-Grundlagen der hier benutzten Betrachtungsweise, die Wahl der unendlich verdünnten Lösung als Normalzustand und die Abzählung der Gruppen gelöster Moleküle, haben nur dann einen Sinn, wenn das LM eindeutig definiert ist, was durch die obige Festsetzung erreicht wird. Allerdings kann man bei streng regulären Lösungen leicht die Funktionen für den gesamten Konzentrationsbereich ermitteln, indem man die Rolle des LM und der gelösten Substanz vertauscht und einmal etwa die Lösung von $\mathrm{A}$ in $\mathrm{B}$, dann die von $\mathrm{B}$ in $\mathrm{A}$ betrachtet und evtl. im mittleren Gebiet interpoliert, wie dies in anderem Zusammenhang schon Fowler und Rushb r o o k e ${ }^{11}$ getan haben.

In der erwähnten Beschränkung liegt zweifellos ein Nachteil der hier entwickelten Methode gegenüber dem quasichemischen und dem Betheschen Verfahren. Andererseits können nach dieser Methode Systeme behandelt werden, bei denen die beiden anderen völlig versagen. Dies gilt nicht nur hinsichtlich der zwischenmolekularen Kräfte, sondern auch im Hinblick auf die Molekülgestalt und die Zahl der Komponenten.

Wie weit man das Näherungsverfahren von II d fortsetzen muß, um einen vorgegebenen Konzentrationsbereich zu erfassen, kann (ebenso wie die Frage, ob die beiden ersten Näherungsprinzipien hier noch gültig sind) nur für den einzelnen Fall entschieden werden; es hängt im wesentlichen von dem Charakter der lokalen Verteilungsfunktionen $\Phi_{r}$ ab. Wir wollen im folgenden die Formeln bis zur II. Näherung entwickeln, um die Methode und ihre Anwendung auf konkrete Fälle zu veranschaulichen.

f) Entwicklung bis zur II. Näherung

Wir haben Zweier- und Dreiergruppen zu betrachten. Die zugehörigen $x_{r}$ unterscheiden wir durch die Indizes $i, i^{\prime}, i^{\prime \prime}$, je nachdem, ob gleiche oder verschiedene Moleküle die Gruppe bilden. Für die Auswertung der Größen $x_{r}$. benötigen wir 
noch die Symmetriezahlen $\sigma_{r}$ jeder Gruppe, die in Tab. 1 zusammengestellt sind.

Aus Gl. (42) ergibt sich zunächst für $h_{i}$ als erste Näherung

$$
h_{i}=N_{1 i} / N_{0},
$$

als zweite

$h_{i}=N_{1 i} / N_{0}\left(1-2 \varkappa_{i i} N_{1 i} / N_{0}-\sum_{i^{\prime}}^{\prime} \varkappa_{i i^{\prime}} N_{1 i^{\prime}} / N_{0}^{*}\right)$,

wo die Summe über alle $i$ mit Ausnahme desjenigen, auf das sich $h_{i}$ bezieht, zu erstrecken ist. Für

$\begin{array}{ll}\text { Gruppe } & \sigma_{r} \\ i i & 2 \\ i i^{\prime} & 1 \\ i i i & 6 \\ i i i^{\prime} & 2 \\ i i^{\prime} i^{\prime \prime} & 1\end{array}$

Tab. 1. Symmetriezahlen.

die I. Näherung lautet die Gl. (43)

$$
\frac{\partial g}{\partial x_{i i}}=\left(N_{1 i} / N_{0}\right)^{2}, \quad \frac{\partial g}{\partial x_{i i^{\prime}}}=N_{1 i} N_{1 i^{\prime}} / N_{0}^{2}
$$

In II. Näherung haben wir das System

$\frac{\partial g}{\partial x_{i i}}=\left(N_{1 i} / N_{0}\right)^{2}\left(1-2 \varkappa_{i i} N_{1 i} / N_{0}-\sum_{i^{\prime}}^{\prime} \varkappa_{i i^{\prime}} N_{1 i^{\prime}} / N_{0}\right)^{2}$

$\frac{\partial g}{\partial x_{i i^{\prime}}}=\left(N_{1 i} N_{1 i^{\prime}} / N_{0}^{2}\right)\left(1-2 \varkappa_{i i} N_{1 i} / N_{0}-\sum_{i^{\prime}}^{\prime} \varkappa_{i i^{\prime}} N_{1 i^{\prime}} / N_{0}\right)\left(1-2 \varkappa_{i^{\prime} i^{\prime}} N_{1 i^{\prime}} / N_{0}-\sum_{i}^{\prime} \varkappa_{i^{\prime} i} N_{1 i} / N_{0}\right)$,

$\frac{\partial g}{\partial x_{i i i}}=\left(N_{1 i} / N_{0}\right)^{3}\left(1-2 \varkappa_{i i} N_{1 i} / N_{0}-\sum_{i^{\prime}}^{\prime} \varkappa_{i i^{\prime}} N_{1 i^{i}} / N_{0}\right)^{3}$

$\frac{\partial g}{\partial x_{i i i^{\prime}}}=\left(N_{1 i} / N_{0}\right)^{2}\left(N_{1 i^{\prime}} / N_{0}\right)\left(1-2 \varkappa_{i i} N_{1 i} / N_{0}-\sum_{i^{\prime}}^{\prime} \varkappa_{i i^{\prime}} N_{1 i^{\prime}} / N_{0}\right)^{2}\left(1-2 \varkappa_{i^{\prime} i^{\prime}} N_{1 i^{\prime}} / N_{0}-\sum_{i}^{\prime} \varkappa_{i^{\prime} i} N_{1 i} / N_{0}\right)$,

$\frac{\partial g}{\partial x_{i i^{\prime} i^{\prime \prime}}}=\left(N_{1 i} N_{1 i^{\prime}} N_{1 i^{\prime \prime}} / N_{0}^{3}\right)\left(1-2 \varkappa_{i i} N_{1 i} / N_{0}-\sum_{i^{\prime}}^{\prime} \varkappa_{i i^{\prime}} N_{1 i^{\prime}} / N_{0}\right)\left(1-2 \varkappa_{i^{\prime} i^{\prime}} N_{1 i^{\prime}} / N_{0}-\sum_{i}^{\prime} \varkappa_{i^{\prime} i} N_{1 i} / N_{0}\right)$

$$
\left(1-2 \varkappa_{i^{\prime \prime} i^{\prime \prime}} N_{1 i^{\prime \prime}} / N_{0}-\sum_{i}^{\prime} \varkappa_{i^{\prime \prime} i} N_{1 i} / N_{0}\right) \text {. }
$$

Bei der Integration ist zu beachten, daß jeweils das Glied mit $x_{i} \ldots i$ die höchste Potenz von $N_{1^{i}} / N_{0}$ festlegt, die berücksichtigt werden darf. Alle höheren sind für die betreffende Näherung physikalisch sinnlos und entfallen. Die Lösung des Systems (45a) lautet dann

$$
\begin{aligned}
& g=\sum_{i} \varkappa_{i i}\left(N_{1 i} / N_{0}\right)^{2}+\sum_{i}^{\prime} \sum_{i^{\prime}}^{\prime} \cdot \varkappa_{i i^{\prime}} N_{1 i} N_{1 i^{\prime}} / N_{0}^{2}+\sum_{i}\left(N_{1 i} / N_{0}\right)^{3}\left(\varkappa_{i i i}-2 \varkappa_{i i}^{2}\right) \\
& \quad+\sum_{i}^{\prime} \sum_{i^{\prime}}^{\prime}\left(N_{1 i}^{2} N_{1 i^{\prime}} / N_{0}^{3}\right)\left(\varkappa_{i i i^{\prime}}-2 \varkappa_{i i} \varkappa_{i i^{\prime}}-\frac{1}{2} \varkappa_{i i^{\prime}}^{2}\right)+\sum_{i}^{\prime} \sum_{i^{\prime}}^{\prime} \sum_{i^{\prime \prime}}^{\prime}\left(N_{1 i} N_{1 i^{\prime}} N_{1 i^{\prime \prime}} / N_{0}^{3}\right)\left(\varkappa_{i i^{\prime} i^{\prime \prime}}-\varkappa_{i i^{\prime}} \varkappa_{i^{\prime} i^{\prime \prime}}\right) .
\end{aligned}
$$

Aus Gl. (30) und (46) folgt mit Benutzung von Gl. (15) und (28), wenn wir in dieser Näherung $\Lambda_{r}^{\prime} /\left(N_{1 i}+\Lambda_{i}\right) \approx 1$ setzen

$\ln B(T)=-E_{0} / k T+\left(N_{2}+\sum_{i} n_{i} N_{1 i}\right) \ln \Gamma_{1}+\sum_{i} N_{1 i} \ln \left(N_{1 i}+\Lambda_{i}\right)+\sum_{i} \Lambda_{i} \ln \left[\left(N_{1 i}+\Lambda_{i}\right) / \Lambda_{i}\right]$

$$
\begin{aligned}
& +N_{2} \ln N_{2}-\sum_{i} N_{1 i}-N_{2}+\frac{1}{2} \sum_{i} \sum_{i^{\prime}} N_{1 i} \gamma_{i^{\prime}} \Phi_{i i^{\prime}}+\frac{1}{6} \sum_{i} \sum_{i^{\prime}} \sum_{i^{\prime \prime}} N_{1 i} \gamma_{i^{\prime}} \gamma_{i^{\prime \prime}} \Phi_{i i^{\prime} i^{\prime \prime}} \\
& -\frac{1}{2} \sum_{i} \sum_{i^{\prime}} \sum_{i^{\prime \prime}} N_{1 i} \gamma_{i^{\prime}} \gamma_{i^{\prime \prime}} \Phi_{i i^{\prime}} \Phi_{i^{\prime} i^{\prime \prime}},
\end{aligned}
$$

wo jetzt die Summierung über alle Werte von $i, i^{\prime}, i^{\prime \prime}$ (einschließlich $i=i^{\prime}$ usw.) geht und $\Phi_{i i^{\prime}}$ auch den Fall $\Phi_{i i}$ einschließt usw. Diese Form gibt noch nicht die reine Potenzreihendarstellung der Solva- 
tation ${ }^{28}$. Um sie zu erhalten, ist es notwendig, auch die $\gamma_{i}$ noch zu entwickeln. Dabei haben wir konsequenterweise nur die Störung I. Ordnung zu berücksichtigen. Aus Gl. (14) und (15) folgt dann (wenn $\sum_{i^{\prime}} n_{i^{\prime}} \varrho_{i^{\prime}} N_{1 i^{\prime}}$ gegen $\frac{1}{2} \sum_{i^{\prime}} a_{i i^{\prime}} n_{i^{\prime}} N_{1 i^{\prime}}$ vernachlässigt wird ${ }^{29}$ )

$$
\gamma_{i^{\prime}}=\frac{1}{f(z)_{i^{\prime}}} \frac{N_{1 i^{\prime}}}{N_{2}+\sum_{i} n_{i} N_{1 i}}\left(1+\frac{1}{2} \sum_{i^{\prime \prime}} \alpha_{i^{\prime} i^{\prime \prime}} n_{i^{\prime \prime}} \frac{N_{1 i^{\prime \prime}}}{N_{2}+\sum_{i} n_{i} N_{1 i}}\right)
$$
und wir erhalten, wenn wir den athermischen Anteil von $\ln B(T)$ zur Abkürzung mit $\ln B(T)_{\text {ath }}$ be-
zeichnen,

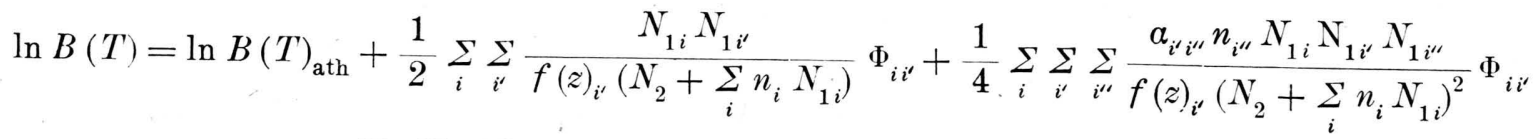

$$
\begin{aligned}
& +\frac{1}{6} \sum_{i} \sum_{i^{\prime}} \sum_{i^{\prime \prime}} \frac{N_{1 i} N_{1 i^{\prime \prime}} N_{1 i^{\prime \prime}}}{f(z)_{i^{\prime}}} \frac{1}{f(z)_{i^{\prime \prime}}\left(N_{2}+\sum_{i} n_{i} N_{1 i}\right)^{2}} \Phi_{i i^{\prime} i^{\prime \prime}}-\frac{1}{2} \sum_{i} \sum_{i^{\prime}} \sum_{i^{\prime \prime}} \frac{N_{1 i} N_{1 i^{\prime}} N_{1 i^{\prime \prime}}}{f(z)_{i^{\prime}} f(z)_{i^{\prime \prime}}\left(N_{2}+\sum_{i} n_{i} N_{1 i}\right)^{2}} \Phi_{i i^{\prime}} \Phi_{i^{\prime} i^{\prime \prime}} .
\end{aligned}
$$

\section{Ubergang zur Thermodynamik. Einige Spezialfälle}

a) Ableitung der thermodynamischen Formeln

Aus Gl. (49) erhält man die Helmholtzsche freie Energie mittels der Gleichung

$$
A_{L}^{0}=-k T\left[\sum_{i} N_{1 i}\left(\ln \frac{G_{1 i}}{N_{1 i}}+1\right)+N_{2}\left(\ln \frac{G_{2}}{N_{2}}+1\right)+\ln B(T)\right]
$$

und das thermodynamische Potential der Lösung aus

$$
F_{L}=A_{L}^{0}+V_{0} p\left(1-\frac{1}{2} \varkappa p\right)
$$

( $V_{0}=$ Volumen des Systems, wie $A_{L}^{0}$ auf den Druck $p \approx 0$ bezogen, $\varkappa=$ Kompressibilität). Alles weitere ergibt sich durch rein thermodynamische Rechnung.

b) Streng reguläre binäre Lösung

Wir betrachten eine Mischung zweier Substanzen, deren Moleküle kugelförmig und von gleicher Größe seien. Das Potential der zwischenmolekularen Kräfte sei für beide kugelsymmetrisch, so daß die Energie in Paaren additiv ist.

Hier hat $B(T)_{\text {ath }}$ den Wert der idealen Lösung, es ist $\alpha_{i}=0, n_{i}=1$ und $f(z)_{i}=1$. Bezeichnet $\varepsilon_{2}$ die Wechselwirkungsenergie eines Paares (bezogen auf $E_{0}$ ), so ist die lokale Verteilungsfunktion der Zweiergruppen

$$
\Phi_{2}=z\left[\exp \left(-\varepsilon_{2} / k T\right) I_{2} / \Gamma_{1}-1\right] .
$$

Setzen wir noch voraus, daß die nächsten Nachbarn eines Gitterpunktes nicht selbst nächste Nachbarn sind, so gilt ferner für die Dreiergruppen

$$
\Phi_{3}=2 z(z-1)\left[\exp \left(-2 \varepsilon_{2} / k T\right)\left(\Gamma_{2} / \Gamma_{1}\right)^{2}-1\right] \text {. }
$$

Damit wird, wenn wir $E_{0}=\frac{z}{2}\left(N_{1} \varepsilon_{11}+N_{2} \varepsilon_{22}\right)$ und $\Gamma_{1}^{N_{1}+N_{2}}=v_{1}^{N_{1}} v_{2}^{{ }^{N_{2}}}$ setzen.

28 Die athermischen Glieder werden zweckmäßig erst in den thermodynamischen Formeln entwickelt.

29 Bei hochpolymeren Kugelmolekülen ist dies nicht zulässig. 


$$
\begin{aligned}
F_{L}= & k T\left\{N_{1} \ln \frac{N_{1}}{N_{1}+N_{2}}+N_{2} \ln \frac{N_{2}}{N_{1}+N_{2}}-N_{1} \ln G_{1} v_{1}-N_{2} \ln G_{2} v_{2}\right. \\
& +\frac{z}{2} \frac{N_{1} \varepsilon_{11}+N_{2} \varepsilon_{2}}{k T}-\frac{z}{2} \frac{N_{1}^{2}}{N_{1}+N_{2}}\left[\exp \left(-\varepsilon_{2} / k T\right) I_{2} / I_{1}-1\right] \\
& -\frac{z}{3}(z-1) \frac{N_{1}^{3}}{\left(N_{1}+N_{2}\right)^{2}}\left[\exp \left(-2 \varepsilon_{2} / k T\right)\left(I_{2} / I_{1}\right)^{2}-1\right] \\
& \left.\quad \frac{z^{2}}{2} \frac{N_{1}^{3}}{\left(N_{1}+N_{2}\right)^{2}}\left[\exp \left(-2 \varepsilon_{2} / k T\right)\left(I_{2} / I_{1}\right)^{2}-2 \exp \left(-\varepsilon_{2} / k T\right) I_{2} / I_{1}+1\right]\right\}
\end{aligned}
$$

Dabei haben wir der Einfachheit halber $A_{L}^{0}=F_{L}$ gesetzt, da das Druckglied hier ohne Interesse und auch praktisch meistens ohne Bedeutung ist. Aus (53) folgt für die freie Energie der Verdünnung mit Gl. (16) und $\varepsilon_{2} / k=E_{2} / R$

$\Delta \mu_{2}=R T\left\{\ln \left(1-s_{1}\right)+s_{1}^{2} \frac{z}{2}\left[\exp \left(-E_{2} / R T\right) \Gamma_{2} / \Gamma_{1}-1\right]+s_{1}^{3} \frac{z}{3}(z-1)\right.$

$\left.\cdot\left[\exp \left(-2 E_{2} / R T\right)\left(\Gamma_{2} / \Gamma_{1}\right)^{2}-1\right]-s_{1}^{3} \frac{z^{2}}{2}\left[\exp \left(-2 E_{2} / R T\right)\left(\Gamma_{2} / \Gamma_{1}\right)^{2}-2 \exp \left(-E_{2} / R T\right) \Gamma_{2} / I_{1}+1\right]\right\}$

und hieraus für die Verdünnungswärme

$$
\begin{aligned}
\Delta H_{2}=- & \frac{z}{2} E_{2} \exp \left(-E_{2} / R T\right)\left(\Gamma_{2} / I_{1}\right) s_{1}^{2} \\
& -\left\{\left[\frac{2}{3} z(z-1)-z^{2}\right] E_{2} \exp \left(-2 E_{2} / R T\right)\left(\Gamma_{2} / \Gamma_{1}\right)^{2}+z^{2} E_{2} \exp \left(-E_{2} / R T\right) I_{2} / I_{1}\right\} N_{1}^{3} .
\end{aligned}
$$

Wenn $E_{2} \ll k T$ ist, können wir die Exponentialfunktionen bis zum kubischen Glied entwickeln und erhalten dânn, wenn wir noch $\Gamma_{2} \approx \Gamma_{1}$ setzen.

$$
\begin{aligned}
\Delta_{H_{2}}= & -\frac{z}{2} E_{2}\left[1-E_{2} / R T+\frac{1}{2}\left(E_{2} / R T\right)^{2}-\frac{1}{6}\left(E_{2} / R T\right)^{3}\right] N_{1}^{2}-\left\{\frac { 2 } { 3 } z ( z - 1 ) E _ { 2 } \left[1-2 E_{2} / R T\right.\right. \\
& \left.\left.+2\left(E_{2} / R T\right)^{2}-\frac{4}{3}\left(E_{2} / R T\right)^{3}\right]+z^{2} E_{2}\left[E_{2} / R T-\frac{3}{2}\left(E_{2} / R T\right)^{2}+\frac{7}{6}\left(E_{2} / R T\right)^{3}\right]\right\} N_{1}^{3} \cdot(55 \mathrm{a})
\end{aligned}
$$

Die Reihenentwicklung nach der Betheschen und quasichemischen Methode wurde von O r $\mathrm{r}^{6}$ angegeben. Sie lautet in unserer Schreibweise

$\Delta_{H_{2}}=-\frac{z}{2} E_{2}\left[1-E_{2} / R T-\frac{1}{2}\left(E_{2} / R T\right)^{3}\right] N_{1}^{2}-\frac{z}{2} E_{2}\left[4 E_{2} / R T+6\left(E_{2} / R T\right)^{3}\right] N_{1}^{3}$.

Man sieht, daß für kleine $E_{2}$ und $N_{1}$ (I. Näherung) beide Theorien zu dem gleichen Ergebnis führen, dảs bereits über die Ansätze von $\mathrm{He}$ it le ${ }^{30}$, $\mathrm{Hildebrand}{ }^{1}$ und $\mathrm{Sc}$ at char d ${ }^{31}$ hinausgeht, welehe gewissermaßen die nullte Näherung darstellen. Bei den höheren Gliedern zeigen sich starke Unterschiede, deren physikalisch wichtigster darin besteht, daß nach O r r das Vorzeichen derselben unabhängig von dem Vorzeichen der Wechselwirkungsenergie $E_{2}$ ist. Bemerkenswert ist ferner die Tatsache, daß in Gl. (55a) auch die höheren Potenzen von $\boldsymbol{N}_{\mathbf{1}}$ Glieder mit $E_{2}$ enthalten, während in Gl. (55 b) das niedrigste Glied des kubischen Terms proportional $E_{2}^{2} / R T$ ist. Dies hat zur Folge, daß für $E_{2} \ll R T$ das in $x_{1}$ quadratische Glied hier bei weitem überwiegt.

Wir wollen nun an dem einfachen Beispiel der streng regulären Lösung noch kurz die Bedeutung der Funktionen $\Gamma$ erläutern. Dazu wählen wir für die zwischenmolekularen Schwingungen das Ein -

30 W. H e i t le r, Ann. Physik 80,630 [1926].

31 G. S c a t c h a rd, Chem. Reviews 8, 321 [1931]. 
st e i n sche Modell der Planckschen Oszillatoren. Dann wird ( $h=$ Plancksches Wirkungsquantum, $\nu=$ Frequenz)

$$
\Gamma_{1}=\left[1-\exp \left(-\frac{h v_{1}}{k T}\right)\right]^{-3} \approx\left(\frac{h v_{1}}{k T}\right)^{-3}, \quad \Gamma_{2} \approx\left(\frac{h v_{2}}{k T}\right)^{-3} .
$$

Setzen wir $\nu_{2}=\nu_{1}+\Delta \nu$, so haben wir in den lokalen Verteilungsfunktionen Exponenten der Form

$$
\frac{E_{2}+3 k T \ln \frac{\nu_{1}+\Delta v}{\nu_{1}}}{k T} \approx \frac{E_{2}+3 k T \frac{\Delta \nu}{\nu_{1}}}{k T} .
$$

Der Ausdruck $\Gamma_{2} / \Gamma_{1}$ ist somit temperaturunabhängig, was wir oben schon benutzt haben. Die Bedingung für die bisher benutzte Näherung lautet

$$
E_{2} \gg k T \frac{\Delta v}{v_{1}} .
$$

Dieses Ergebnis lälst sich auch aus der Betrachtung eines einzelnen Oszillators ableiten, wenn man $E_{2}$ als Änderung der potentiellen Energie der Ruhelage auffaßt und beachtet, daß $k T$ die mittlere statistische Energie eines Oszillators ist ${ }^{27}$.

$$
\text { c) Monodisperse Lösung hochpolymerer Fadenmoleküle }
$$

Dieses Problem ist an anderer Stelle ${ }^{32}$ ausführlich behandelt worden. Wir können uns daher hier auf die Wiedergabe der wichtigsten Formeln beschränken. Man erhält für die freie Energie der Verdïnnung

$$
\begin{aligned}
\Delta \mu_{2}=-R T_{1}\{1+ & \frac{1}{2}\left[a_{n} n-\frac{1}{f(z)} \Phi_{2}\right] x_{1} \\
& \left.+\frac{1}{2}\left[2 \beta n^{2}+\frac{1}{2} a_{n}^{2} n^{2}-\frac{1}{2 f(z)} a_{n} n \Phi_{2}+\frac{2}{f(z)^{2}} \Phi_{2}^{2}-\frac{2}{3 f(z)^{2}} \Phi_{3}\right] x_{1}^{2}\right\},
\end{aligned}
$$

für die Verdünnungsentropie

$$
\begin{aligned}
\Delta s_{2}=R s_{1} & \left\{1+\frac{1}{2}\left[a_{n} n-\frac{1}{f(z)}\left(\Phi_{2}+T \frac{\partial \Phi_{2}}{\partial T}\right)\right] N_{1}+\frac{1}{2}\left[2 \beta n^{2}+\frac{1}{2} a_{n}^{2} n^{2}\right.\right. \\
& \left.\left.-\frac{1}{2 f(z)} a_{n} n\left(\Phi_{2}+T \frac{\partial \Phi_{2}}{\partial T}\right)+\frac{2}{f(z)^{2}}\left(\Phi_{2}^{2}+2 T \Phi_{2} \frac{\partial \Phi_{2}}{\partial T}\right)-\frac{2}{3 f(z)^{2}}\left(\Phi_{3}+T \frac{\partial \Phi_{3}}{\partial T^{T}}\right)\right] N_{1}^{2}\right\},
\end{aligned}
$$

für die Verdünnungswärme

$$
\Delta_{H_{2}}=-\frac{R T^{2}}{2 f(z)} N_{1}^{2}\left\{\frac{\partial \Phi_{2}}{\partial T}+\left[\frac{1}{2} a_{n} n \frac{\partial \Phi_{2}}{\partial T}-\frac{4}{f(z)} \Phi_{2} \frac{\partial \Phi_{2}}{\partial T^{\prime}}+\frac{2}{3 f(z)} \frac{\partial \Phi_{3}}{\partial T}\right] N_{1}\right\},
$$

wo $\alpha_{n}$ der Störungsparameter und $\beta$ der Koeffizient des linearen Gliedes in der Entwicklung der „,Störungsfunktion" $\alpha(\ldots)$ ist.

d) Polydisperse Lösung hochpolymerer Fadenmoleküle

In diesem Falle unterscheiden sich die gelösten Komponenten nur durch den Polymerisationsgrad $n$, den wir daher als Index an Stelle von $i$ einführen. Wir definieren ferner die Verteilungsfunktion der Polymerisationsgrade durch die Gleichung

$$
N_{1 n}=N_{1} f(n)
$$

32 A. II ü n st e r, Kolloid-Z. (im Druck). 
wo $N_{1}$ die gesamte Molekülzahl der Fadenmoleküle ist. Wir können dann die einzelnen Summanden der Gl. (49) als Funktionen der stetig veränderlichen Größen $n, n^{\prime}, n^{\prime \prime}$ auffassen und die Summen durch Integrale ersetzen. Definieren wir den mittleren Polymerisationsgrad $\bar{n}$ durch die Gleichung

so nimmt Gl. (49) die Gestalt an:

$$
\bar{n}=\int_{0}^{\infty} n f(n) d n
$$

$\ln B(T)=\ln B(T)_{\text {ath }}+\frac{1}{2} \frac{N_{1}^{2}}{N_{2}+\bar{n} N_{1}} \int_{0}^{\infty} \int_{0}^{\infty} f(n) \frac{f\left(n^{\prime}\right)}{f(z)_{n^{\prime}}} \Phi_{n n^{\prime}} d n d n^{\prime}$

$$
\begin{aligned}
& \left.+\frac{1}{4} \cdot \frac{N_{1}^{3}}{\left(N_{2}+\bar{n}\right.} N_{1}\right)^{2} \cdot \int_{0}^{\infty} \int_{0}^{\infty} \int_{0}^{\infty} f(n) \alpha_{n^{\prime}} \frac{f\left(n^{\prime}\right)}{f(z)_{n^{\prime}}} n^{\prime \prime} f\left(n^{\prime \prime}\right) \Phi_{n n^{\prime}} d n d n^{\prime} d n^{\prime \prime} \\
& \left.+\frac{1}{6} \frac{N_{1}^{3}}{\left(N_{2}+\bar{n}\right.} N_{1}\right)^{2} \int_{0}^{\infty} \int_{0}^{\infty} \int_{0}^{\infty} f(n) \frac{f\left(n^{\prime}\right)}{f(z)_{n^{\prime}}} \frac{f\left(n^{\prime \prime}\right)}{f(z)_{n^{\prime \prime}}} \Phi_{n n^{\prime} n^{\prime \prime}} d n d n^{\prime} d n^{\prime \prime} \\
& -\frac{1}{2} \frac{N_{1}^{3}}{\left(N_{2}+\bar{n} N_{1}\right)^{2}} \int_{0}^{\infty} \int_{0}^{\infty} \int_{0}^{\infty} f(n) \frac{f\left(n^{\prime}\right)}{f(z)_{n^{\prime}}} \frac{f\left(n^{\prime \prime}\right)}{f(z)_{n^{\prime \prime}}} \Phi_{n n^{\prime}} \Phi_{n^{\prime} n^{\prime \prime}} d n d n^{\prime} d n^{\prime \prime} \text {. }
\end{aligned}
$$

Daraus folgt mit Gl. (51) für die freie Energie der Verdünnung $\left(N_{1}=\frac{N_{1}}{N_{2}+\bar{n} N_{1}}\right)$

$$
\begin{aligned}
\Delta \mu_{2}= & -R T \int_{N_{1}}+\frac{1}{2}\left[\int_{0}^{\infty} \int_{0}^{\infty} a_{n} n^{\prime} f(n) f\left(n^{\prime}\right) d n d n^{\prime}-\int_{0}^{\infty} \int_{0}^{\infty} f(n) \frac{f\left(n^{\prime}\right)}{f^{\prime}(z)_{n^{\prime}}} \Phi_{n n^{\prime}} d n d n^{\prime}\right] N_{1}^{2} \\
& +\frac{1}{2}\left[\int_{0}^{\infty} \int_{0}^{\infty} \int_{0}^{\infty}\left(2 \beta+\frac{1}{2} a_{n}\right) n^{\prime} n^{\prime \prime} f(n) f\left(n^{\prime}\right) f\left(n^{\prime \prime}\right) d n d n^{\prime} d n^{\prime \prime}\right. \\
& -\frac{1}{2} \int_{0}^{\infty} \int_{0}^{\infty} \int_{0}^{\infty} f(n) a_{n^{\prime}} \frac{f\left(n^{\prime}\right)}{f(z)_{n^{\prime}}} n^{\prime \prime} f\left(n^{\prime \prime}\right) \Phi_{n n^{\prime}} d n d n^{\prime} d n^{\prime \prime} \\
& +\int_{0}^{\infty} \int_{0}^{\infty} \int_{0}^{\infty} f(n) \frac{f\left(n^{\prime}\right)}{f(z)_{n^{\prime}}} \frac{f\left(n^{\prime \prime}\right)}{f(z)_{n^{\prime \prime}}} \Phi_{n n^{\prime}} \Phi_{n^{\prime} n^{\prime \prime}} d n d n^{\prime} d n^{\prime \prime} \\
& \left.\left.-\frac{1}{3} \int_{0}^{\infty} \int_{0}^{\infty} \int_{0}^{\infty} f(n) \frac{f\left(n^{\prime}\right)}{f(z)_{n^{\prime}}} \frac{f\left(n^{\prime \prime}\right)}{f(z)_{n^{\prime \prime}}} \Phi_{n n^{\prime} n^{\prime \prime}} d n d n^{\prime} d n^{\prime \prime}\right] N_{1}^{3}\right] .
\end{aligned}
$$

Es ergibt sich somit, daß die thermodynamischen Eigenschaften der Lösungen hochpolymerer Fadenmoleküle im allgemeinen von der Polydispersität abhängen. Die Verteilungsfunktion $f(n)$ kann in gewissen Fällen theoretisch berechnet und allgemein experimentell (aus Fraktionierungs- oder Sedimentationsversuchen) ermittelt werden. Im letzteren Falle dürfte zu ihrer analytischen Formulierung die von $\mathrm{Ju} 1 \mathrm{l}$ a n d e r ${ }^{33}$

33 I. Jullander, Ark. Kemi Mineral. Geol., Ser. A, 21, Nr. 8 [1945]. eingeführte dreiparametrige Funktion gute Dienste leisten.

In einem Spezialfall läßt sich allerdings zeigen, daß zum mindesten die I. Näherung (der in $s_{1}$ quadratische Term von $\Delta \mu_{2}$ ) nur von dem durch Gl. (62) definierten mittleren Polymerisationsgrad, aber nicht von den Einzelheiten der Verteilung abhängt (für die athermische Lösung wurde dies schon früher nachgewiesen ${ }^{34}$ ). Nimmt man ${ }^{34}$ A. U ü nst e r, Z. Naturforschg. 2a, 272 [1947]. 
nämlich an, daß die Fadenmoleküle starr sind und $\varepsilon_{2}+k T \Delta \nu / \nu_{1} \equiv \eta_{12}>0$ ist. so gilt

$$
\Phi_{n n^{\prime}}=(z-2) n n^{\prime}\left[\exp \left(-\eta_{2} / k T\right)-1\right] \text {. }
$$

Es ist dann $f(z)=z$, und man erhält als I. Näherung

$$
\begin{aligned}
& \ln B(T)=\ln B(T)_{\text {ath }} \\
& +\frac{1}{2} \frac{\left(\bar{n} N_{1}\right)^{2}}{N_{2}+\bar{n} N_{1}} \frac{z-2}{z}\left[\exp \left(-\eta_{2} / k T\right)-1\right] . \\
& 317 \text { G. V. Schulz, Z. physik. Chem., Abt. A, 176, }
\end{aligned}
$$

Die obigen Voraussetzungen treffen näherungsweise bei dem System Nitrocellulose-Aceton zu. Merkwürdigerweise scheint dies der einzige Fall zu sein, in welchem das Problem experimentell untersucht worden ist. G. V. Schul z ${ }^{35}$ fand in der Tat, daß zwei Produkte von verschiedener Polydispersität, aber gleichem mittlerem Molekulargewicht, den gleichen Verlauf des osmotischen Druckes zeigen.

Der Gesellschaft der Freunde der Universität Heidelberg danke ich für die Gewährung eines Forschungsstipendiums.

\title{
Isotopenverschiebung beim Cadmium durch Ionenwanderung in geschmolzenem Cadmiumchlorid
}

\author{
Von Alfred Klemm, Heinrich Hintenberger und Walter Seelmann-Eggebert \\ Aus dem Kaiser-Wilhelm-Institut für Chemie, Tailfingen \\ (Z. Naturforschg. 3a, 172-176 [1948]; eingegangen am 27. Februar 1948)
}

\begin{abstract}
An der Grenze zwischèn $\mathrm{LiCl}$ und $\mathrm{CdCl}_{2}$ wurde durch elektrolytische Ionenwanderung (Dauer $50 \mathrm{~h}$, Stromdichte $5,5 \mathrm{~A} / \mathrm{cm}^{2}$, Trennstrecke $13 \mathrm{~cm}$, Temperatur $630^{\circ} \mathrm{C}$ ) die Isotopenverteilung beim $\mathrm{Cd}$ zugunsten der leichten Isotope verschoben. Der Trennfaktor der besten Fraktion $\left(47 \mathrm{mg} \mathrm{CdCl}_{2}\right)$ in bezug auf natürliches Cd betrug 1,046 für 1\% Massenunterschied, also 1,5 für die Isotope ${ }^{106} \mathrm{Cd}$ und ${ }^{116} \mathrm{Cd}$. Als Masseneffekt ergab sich $\mu=\ln \left({ }^{i} w / k w\right) / \ln \left({ }^{i} m / k m\right)=-0,067$ und als Vervielfachung (Trennstufenzahl) 67.
\end{abstract}

$\mathrm{I}$

n einer Apparatur aus Supremaxglas (Abb.1), die sich von einer früher beschriebenen ${ }^{1}$ nur dadurch unterschied, daß sie etwas länger war (Trennrohr $25,5 \mathrm{~cm}$ statt $22,5 \mathrm{~cm}$ ), wurde die Kette Anodenkohlen- $\mathrm{CdCl}_{2}-\mathrm{LiCl}-\mathrm{PbCl}_{2}-$ Kathodenkohle bei $630^{\circ} \mathrm{C}$ zwei Tage lang mit $95 \mathrm{~V}$ und $550 \mathrm{~mA}$ elektrolysiert. Als Diaphragma im Trennrohr (4 mm Innendurchmesser) diente wieder Quarzpulver $\mathrm{B}_{2}$ (Korndurchmesser $0,1 \mathrm{~mm}$ ) in loser Packung. Der stromdurchflossene Querschnitt im Trennrohr betrug demnach etwa $0,1 \mathrm{~cm}^{2}$, die Stromdichte 5,5 $\mathrm{A} / \mathrm{cm}^{2}$ und die Verschiebung des Cadmiums relativ zum Chlor etwa $3 \mathrm{~m}$. An der Kathode wurde wieder $\mathrm{PbCl}_{2}$ verwendet, weil kathodisch abgeschiedenes Li das Glas zerstören würde und zudem die Pb-Abscheidung sehr bequem ist. Da eine Anreicherung der leichten CdIsotope beabsichtigt war, wurde die Grenze zwischen $\mathrm{LiCl}$ und $\mathrm{CdCl}_{2}$ im Trennrohr so gelegt, daß

1 A. Klemm, H. Hintenberger u. Ph. Hoernes, Z. Naturforschg. 2a, 245 [1947]. der größere Teil des Trennrohres mit $\mathrm{CdCl}_{2}$ gefüllt war.

Der $42 \mathrm{~cm}$ lange elektrische Ofen hatte $42 \mathrm{~mm}$ Innendurchmesser und bestand diesmal aus nur zwei ineinandergesteckten Supremaxrohren, von denen das innere mit Chromnickelband bewickelt war. Im Dauerbetrieb hielt der Ofen bei einer Leistungsaufnahme von $1,6 \mathrm{KW}$ das $\mathrm{LiCl}$ (Schmp. $614^{\circ} \mathrm{C}$ ) geschmolzen.

Etwas schwierig war bei diesen Trennversuchen immer das Füllen der Apparatur. Nach manchen Vorversuchen hatte sich folgendes Füllverfahren bewährt, das bei dem mitgeteilten Versuch auch im wesentlichen eingehalten werden konnte: Die verschiedenen Niveaus, bis zu denen die Apparatur zu fiillen war, wurden vorher mit Wasser festgestellt und angemerkt. Dann wurde die Apparatur mit Chrom-Schwefelsäure gereinigt und samt der Kathodenkohle bei $650^{\circ} \mathrm{C}$ und $2 \mathrm{~mm} \mathrm{Hg-Druck}$ getrocknet.

Wenn man Stücke von wasserfreiem LiCl, $\mathrm{CdCl}_{2}$ oder $\mathrm{PbCl}_{2}$ aus einer Pulverflasche in ein Reagensglas hrachte, dann schmolz und evakuierte, so stiegen in der Regel bei 100 bis $10 \mathrm{~mm} \mathrm{Hg}$ aus den Schmelzen Gashlasen auf. Die Blasenbildung hörte aber wieder auf und man erhielt schließlich alle drei Schmelzen auch 